\title{
On the Use of Dual Acoustic Radiation Forces to Induce Shear Wave Propagation and Interference Pattern Formation
}

\author{
Kenneth Hoyt ${ }^{1,2,3,4}$, Zaegyoo Hah $^{5}$, Chris Hazard ${ }^{6}$, Kevin J Parker $^{5}$ \\ Departments of ${ }^{1}$ Radiology, ${ }^{2}$ Biomedical Engineering and ${ }^{3}$ Electrical \& Computer Engineering \\ ${ }^{4}$ Comprehensive Cancer Center, University of Alabama at Birmingham, Birmingham, AL USA \\ ${ }^{5}$ Department of Electrical \& Computer Engineering, University of Rochester, Rochester, NY USA \\ ${ }^{6}$ General Electric Global Research, Niskayuna, NY USA
}

\begin{abstract}
An elasticity imaging system that uses a pair of acoustic radiation forces was developed to generate shear wave interference patterns. Ultrasound pulse-echo data and correlation-based techniques were used to estimate material deformation and to characterize the viscoelastic response. Both normal and axicon focal configurations were implemented and studied to assess impact on shear wave generation. Theoretical models were shown in simulation to describe shear wave propagation and interference pattern properties. In a tissuemimicking phantom, experimental results were in agreement with theoretical findings. Experimental results also confirm that shear wave interference patterns can be remotely produced in tissue-mimicking material using dynamic acoustic radiation force excitation. Overall, preliminary results are encouraging and the tissue elasticity imaging system described may prove feasible for viscoelastic property characterization in tissue.
\end{abstract}

\section{INTRODUCTION}

It has been shown that a pair of dynamic mechanical sources vibrating at slightly offset frequencies could produce propagating shear waves that constructively combine to create slowly propagating interference patterns [1]. A spatial analysis of these shear wave interference patterns allows elastic property estimation of the underlying medium [2-5]. In a more recent report, it was mathematically theorized that shear wave interference patterns could be generated using a set of dynamic acoustic radiation forces [6]. This hypothesis was validated soon thereafter using a spatially separated pair of external single element transducers [7]. Given encouraging preliminary results using this setup, a prototype ultrasound system was developed that can both induce shear wave propagation and track underlying tissue deformation for shear wave interference pattern imaging [8,9]. Since this system relies on properly timed acoustic radiation forces from the imaging transducer and not external vibration sources, clinical translation of this promising elasticity imaging modality is more tenable. In this paper, we detail operation of this novel a tissue elasticity imaging system in tissue-mimicking phantom and analyze the effects of normal versus axicon focusing of acoustic radiation forces on shear wave propagation and interference pattern formation. Matched simulation results are presented to verify mathematical models.

\section{MATERIALS AND METHODS}

\section{A. Simulations}

Simulations were developed using Matlab software (Mathworks, Natick, MA, USA). As detailed previously, acoustic radiation force-induced tissue displacements were computed using a Green's function analysis. These displacement fields were then projected onto ultrasound backscattered signals for each time step of shear wave propagation. In order to mimic an integrated ultrasound system for both inducing and tracking shear wave motion, the later was performed using correlation-based speckle tracking algorithms.

The Matlab-based program known as FIELD II [10] simulated both high and low intensity ultrasound pulse transmissions for inducing tissue motion and tracking shear wave propagation across the image plane, respectively. For both configurations, the transducer was modeled as a 128 element linear array with a $5 \mathrm{MHz}$ center frequency. A dual beam (separation distance of $18 \mathrm{~mm}$ ) displacement-inducing transducer configuration was simulated with a focal depth of 25 $\mathrm{mm}$ and for a given f-number for remotely producing shear wave propagation either focused using normal or axicon techniques. Imaging region-of-interests (ROIs) were confined to $18 \mathrm{~mm}$ in width and 10 to $37 \mathrm{~mm}$ in depth to reflect experimental system conditions. Given a $0.25 \mathrm{~ms}$ tone burst (1250 cycles at $5 \mathrm{MHz}$ ), intensities were normalized to a timeaverage intensity value of $1000 \mathrm{~W} / \mathrm{cm}^{2}$. Displacement fields were calculated after normalizing radiation force distributions by area under the curve techniques [6].

A uniform isoechoic scattering phantom was also simulated using FIELD II. Initial scatterer locations represent predisplacement reference positions while displacements determined via viscoelastic Green's function analysis were used to reposition the scatterers and reflect shear wave motion following acoustic radiation force excitation. Scatterer repositions for each RF line were computed for each time step along the beam axis using interpolation techniques. Acoustic radiation forces and subsequent tissue displacements were simulated throughout the ROI for each of the two spatially separated pushing beams. Using a 20 sample kernel, axial motion due to underlying shear wave propagation was estimated as the peak position of a cross-correlation function 
applied to congruent pre- and post-displacement RF data segments. One sample kernel shifting allowed displacement estimation as a function of depth and for each simulated RF data sequence. The standard deviation at each spatial location was computed to visualize shear wave interference patterns.

\section{B. Experiments}

An ultrasound system (Logiq 9, GE Medical Systems, Milwaukee, WI, USA) equipped with a dedicated $5 \mathrm{MHz}$ transrectal transducer was modified to both induce and track shear wave propagation. Imaging ROIs were confined to an 18 $\mathrm{mm}$ lateral width at depths of 10 to $37 \mathrm{~mm}$. For RF lines separated by $0.6 \mathrm{~mm}$, this equated to 31 data sequences in the lateral dimension. Ultrasound beams for acoustic radiation force induction of shear wave propagation were focused at a depth of $25 \mathrm{~mm}$ (using either normal or axicon focal configurations) and located on the outer edges of the ROI. Output intensity (spatial peak temporal average intensity, $I_{S P T A}$ ) for the acoustic radiation force pulse sequences were measured to be $1.6 \mathrm{~W} / \mathrm{cm}^{2}$ and $0.5 \mathrm{~W} / \mathrm{cm}^{2}$ for normal and axicon focusing, respectively.

Scanner imaging sequences were developed to permit shear wave generation and subsequent tracking, Figure 3. The process begins with the system acquiring a reference RF data sequence, then a high-intensity ultrasound tone burst $(0.25 \mathrm{~ms})$

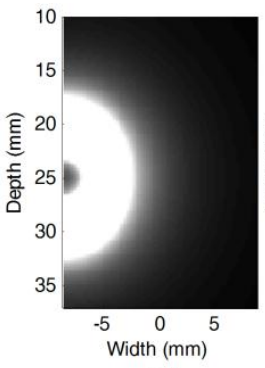

(a)

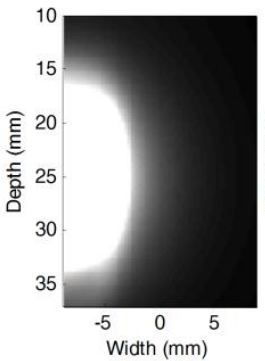

(e)

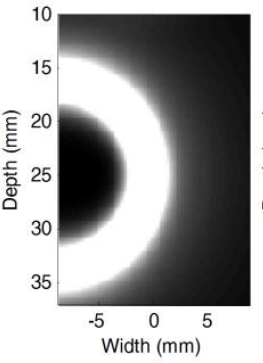

(b)

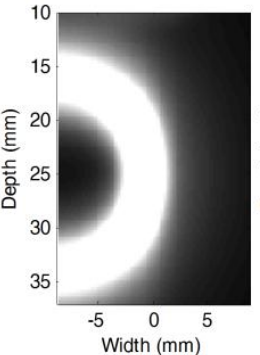

(f)

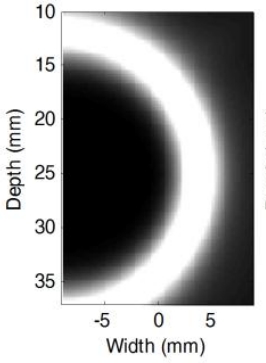

(c)

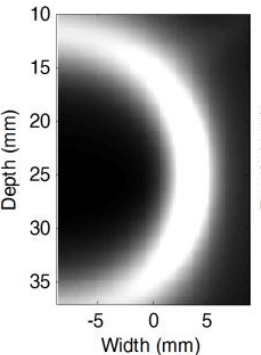

(g)

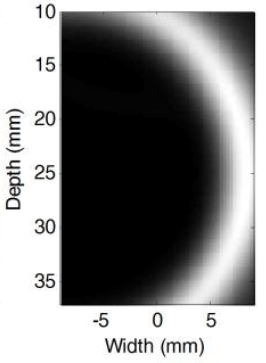

(d)

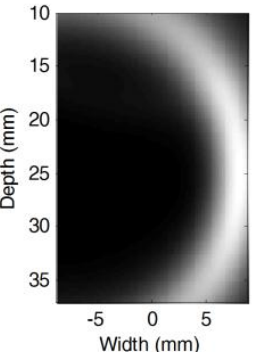

(h)

Figure 1. Simulated shear wave propagation for both normal (top) and axicon (bottom) focusing configurations. After initial displacement, images depict shear waves at time points of (a, e) 1.3, (b, f) 2.5, (c, g) 3.7, and (d, h) 4.9 ms.

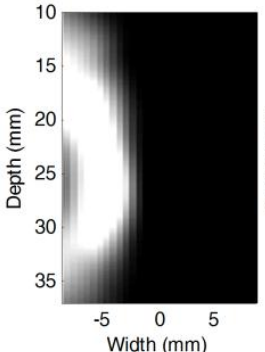

(a)

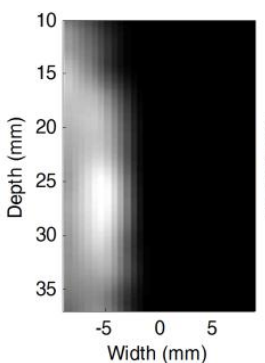

(e)

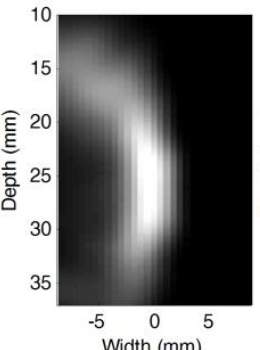

(b)

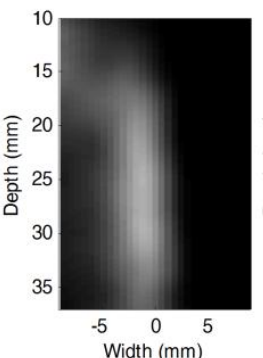

(f)

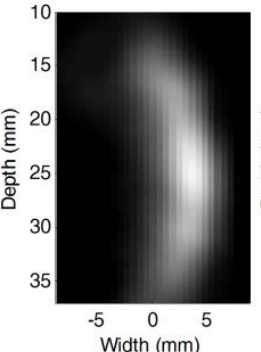

(c)

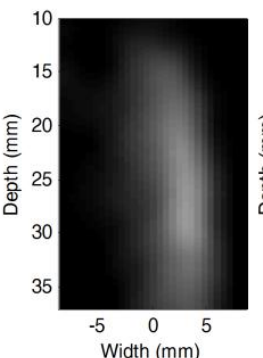

(g)

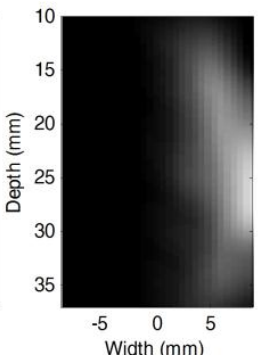

(d)

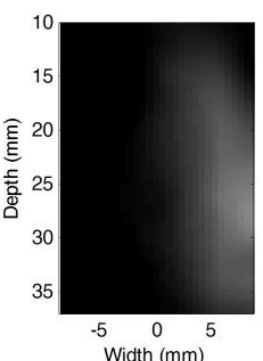

(h)

Figure 2. Experimental shear waves propagation for normal (top) and axicon (bottom) focal configurations. After initial displacement, images depict attenuating shear waves at time points of (a, e) 1.3, (b, f) 2.5, (c, g) 3.7, and (d, h) 4.9 ms. 
induces shear wave propagation. Following a $0.5 \mathrm{~ms}$ delay to minimize reverberation artifacts, 48 additional RF data sequences are collected along the same line of sight at a rate of $2.5 \mathrm{kHz}$. After a short delay, this process is repeated until all 31 lateral data sequences in the ROI are populated. The complete imaging sequence required an elapsed time of $2.4 \mathrm{sec}$. Tissue displacement maps and shear wave interference patterns were then synthesized (at a rate of $500 \mathrm{~Hz}$ ) from the left and right pushing beams. A tissue mimicking phantom was employed for all experiments.

\section{RESULTS}

Attenuating shear waves propagate away from the acoustic focus at a rate equal to the shear wave speed of the supporting medium $(3.6 \mathrm{~m} / \mathrm{s})$, Figure 1. Comparison of simulated images produced using normal or axicon focusing configuration reveal that the former induces propagating shear wave with attenuation at distance. While the shear waves induced using axicon focal characteristics also exhibits noticeable attenuation, the effect is less pronounced in the transverse direction. Since axicon focusing distributes the ultrasound beam energy over a larger spatial area, propagating shear waves are noticeably more planar along the wave fronts. Experimental elasticity results for a single focused ultrasound beam are presented in Figure 2. Similar to the simulation results, attenuated shear waves propagate away from the acoustic radiation force sources. Again, normal focusing produces spherically propagating shear waves whereas axicon focusing yields more planar shear wave fronts. Given that acoustic radiation force induced displacements are on the order of micrometers, displacement estimations can exhibit a low signal-to-noise ratio

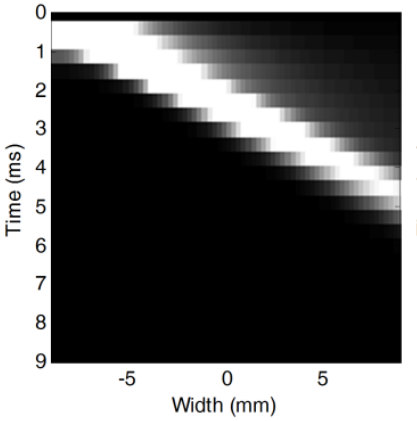

(a)

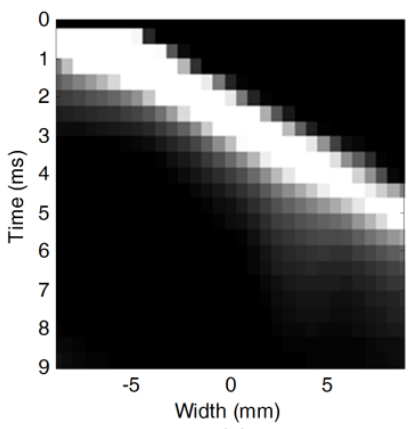

(c)

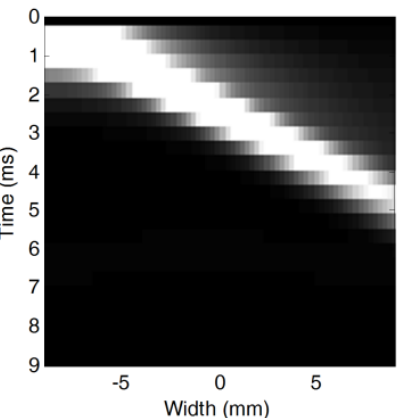

(b)

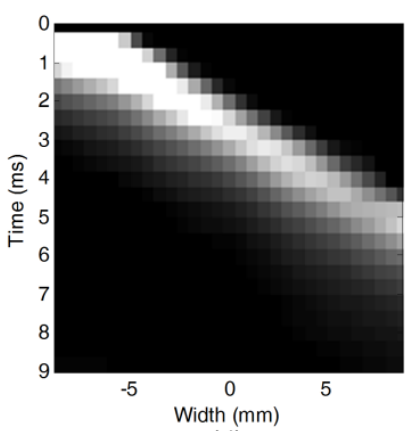

(d)
Figure 3. Temporal characteristics of a propagating shear wave mapped as a function of image plane width. Images detail $(a, b)$ simulated and $(c, d)$ experimental shear wave propagation away from the ultrasound beam focal area for both $(\mathrm{a}, \mathrm{c})$ normal and $(\mathrm{b}, \mathrm{d})$ axicon focal configurations.
(SNR). The cumulative effect of a low SNR is that current image quality for experimental findings are compromised but encouraging.

Temporal characteristics of propagating shear waves induced using acoustic radiation forces mapped as a function of image plane width (from the ultrasound beam focus, $25 \mathrm{~mm}$ ) are presented in Figure 3. Congruence between theoretical and experimental findings is reasonable but inspection of the results reveals some differences. Specifically, experimental shear wave patterns tend to have more variations in the vertical direction and can be slightly wider in the horizontal direction compared with theoretical results. These are likely due to a number of factors including low SNR and small inaccuracies in the numerical model. Temporal characteristics of the propagating shear waves along the transverse dimension (at focal depth) are comparable for both normal and axicon focal configurations. Analysis of each image reveals that the shear wave speed is about $3.6 \mathrm{~m} / \mathrm{s}$.

Application of acoustic radiation forces produce shear waves that propagate away from the initial displacement source. By independently tracking displacement fields across the image plane from two separated sources, periodic tissue motion sequences are produced by delayed superimposition of these same shear wave displacement fields. Given a $500 \mathrm{~Hz}$ repetition frequency for each of the acoustic radiation force

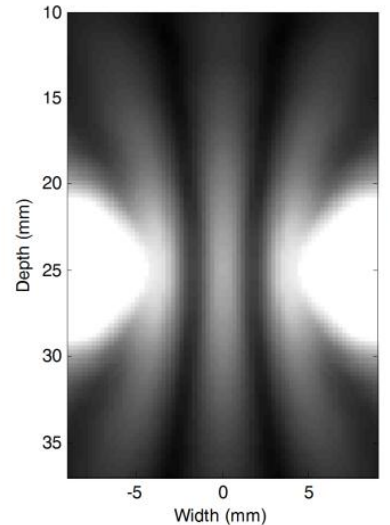

(a)

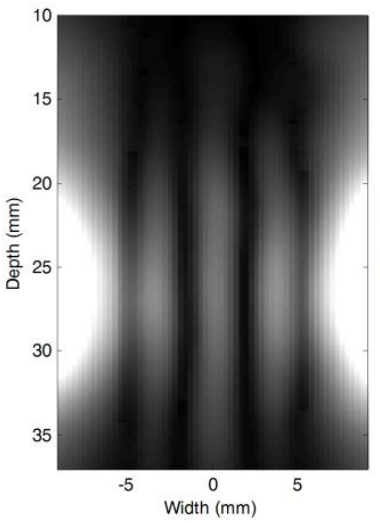

(c)

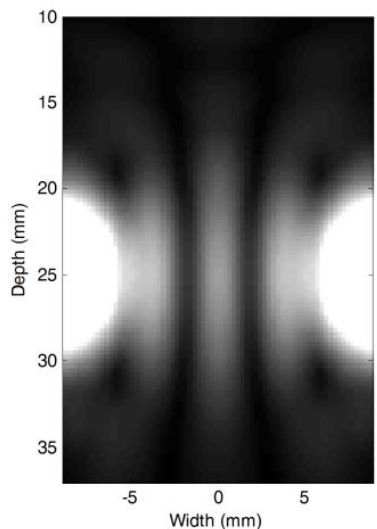

(b)

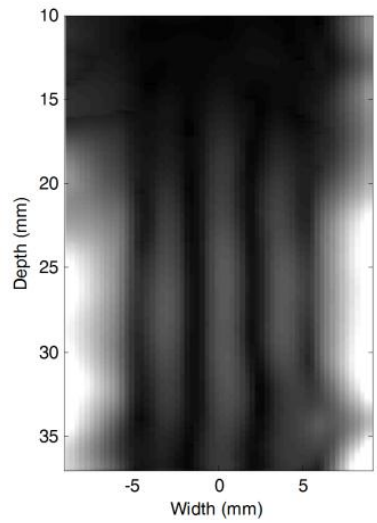

(d)
Figure 4. Shear wave interference pattern images of matched $(a, b)$ simulation and $(c, d)$ experimental findings for both $(a, c)$ normal and $(b, d)$ axicon focal configurations at a depth of $25 \mathrm{~mm}$. 
sources, shear wave interference pattern images were synthesized, Figure 4. Matched simulation and experimental shear wave interference pattern images correspond to either normal or axicon ultrasound beam focusing. Given shear wave interference pattern spatial properties, the shear wave speed of the supporting medium can be estimated as two times the product of the spatial shear wavelength and repetition frequency [1]. For simulated images presented Figure 4, the spatial wavelength at the focal depth was measured to be 3.56 $\mathrm{mm}$, yielding a shear wave speed estimate of $3.56 \mathrm{~m} / \mathrm{s}$ and an error of $1.1 \%$ from the true shear speed (i.e., $3.6 \mathrm{~m} / \mathrm{s}$ ). Likewise, shear wave speed estimates using the experimental shear wave interference pattern images were found to be 3.51 $\mathrm{m} / \mathrm{s}$ and in agreement with those derived from Figure $2(2.5 \%$ error).

Comparison of elasticity images shown in Figure 4 produced using either normal or axicon focal configurations further reveals that normal focusing produces shear wave interference pattern bands in the far-field with discernible spreading and wavelength elongation. If plane wave conditions are of importance, axicon focusing produces better results theoretically although these differences are not as apparent when inspecting experimentally derived shear wave interference pattern images.

\section{DISCUSSION}

Theoretical models were shown in computer simulations to accurately describe experimental findings that were acquired using a modified GE Logiq 9 ultrasound imaging system. The role of ultrasound beam focusing configuration on radiation force induced shear wave propagation was also explored. Although both normal and axicon focusing produced acceptable shear wave interference patterns in terms of image quality, axicon-based results can be achieved at a fraction of the acoustic intensity $\left(0.5 \mathrm{~W} / \mathrm{cm}^{2}\right.$ versus $1.6 \mathrm{~W} / \mathrm{cm}^{2}$ for normal focusing). Given FDA recommendations that ultrasound intensity not exceed $0.72 \mathrm{~W} / \mathrm{cm}^{2}$, axicon focusing presents an advantage compared to the higher intensity outputs associated with normal focusing. Elasticity measurements derived from shear wave interference pattern images were in good agreement with actual values.

\section{CONCLUSIONS}

Experimental validation of acoustic radiation force induced shear wave interference patterns was presented and results warrant further research using this promising imaging modality.

\section{ACKNOWLEDGMENT}

This research project was supported in part by NIH Grant 5RO1AG016317-07.

\section{REFERENCES}

[1] Z. Wu, L. S. Taylor, D. J. Rubens, and K. J. Parker, "Sonoelastographic imaging of interference patterns for estimation of the shear velocity of homogeneous biomaterials," Phys Med Biol, vol. 49, no. 6, pp. 911-22, Mar, 2004.

[2] Z. Wu, K. Hoyt, D. J. Rubens, and K. J. Parker, "Sonoelastographic imaging of interference patterns for estimation of shear velocity distribution in biomaterials," J Acoust Soc Am, vol. 120, no. 1, pp. 53545, Jul, 2006.

[3] K. Hoyt, K. J. Parker, and D. J. Rubens, "Real-time shear velocity imaging using sonoelastographic techniques," Ultrasound Med Biol, vol. 33, no. 7, pp. 1086-97, Jul, 2007.

[4] K. Hoyt, T. Kneezel, B. Castaneda, and K. J. Parker, "Quantitative sonoelastography for the in vivo assessment of skeletal muscle viscoelasticity," Phys Med Biol, vol. 53, no. 15, pp. 4063-80, Aug, 2008.

[5] K. Hoyt, B. Castaneda, and K. J. Parker, "Two-dimensional sonoelastographic shear velocity imaging," Ultrasound Med Biol, vol. 34, no. 2, pp. 276-88, Feb, 2008.

[6] K. Hoyt, "Theoretical analysis of shear wave interference patterns by means of dynamic acoustic radiation forces," Int J Multiphys, vol. 5, no. 1, pp. 9-24, Mar, 2011.

[7] Z. Hah, C. Hazard, Y. T. Cho, D. Rubens, and K. Parker, "Crawling waves from radiation force excitation," Ultrason Imaging, vol. 32, no. 3, pp. 177-89, Jul, 2010.

[8] Z. Hah, C. Hazard, B. Mills, C. Barry, D. Rubens, and K. Parker, "Integration of crawling waves in an ultrasound imaging system. Part 2: signal processing and applications," Ultrasound Med Biol, vol. 38, no. 2, pp. 312-23, Feb, 2012.

[9] C. Hazard, Z. Hah, D. Rubens, and K. Parker, "Integration of crawling waves in an ultrasound imaging system. Part 1: system and design considerations," Ultrasound Med Biol, vol. 38, no. 2, pp. 296-311, Feb, 2012

[10] J. A. Jensen, and N. B. Svendsen, "Calculation of pressure fields from arbitrarily shaped, apodized, and excited ultrasound transducers," IEEE Trans Ultrason Ferroelectr Freq Control, vol. 39, no. 2, pp. 262-7, 1992. 